\title{
Competition between Diffusion and Fragmentation: An Important Evolutionary Process of Nature
}

\author{
Jesper Ferkinghoff-Borg, Mogens H. Jensen, Joachim Mathiesen, Poul Olesen and Kim Sneppen* \\ Niels Bohr Institute, Blegdamsvej 17, DK-2100 Copenhagen Ø, Denmark
}

(Dated: November 10, 2018)

\begin{abstract}
We investigate systems of nature where the common physical processes diffusion and fragmentation compete. We derive a rate equation for the size distribution of fragments. The equation leads to a third order differential equation which we solve exactly in terms of Bessel functions. The stationary state is a universal Bessel distribution described by one parameter, which fits perfectly experimental data from two very different system of nature, namely the distribution of ice crystal sizes from the Greenland ice sheet and the length distribution of $\alpha$-helices in proteins.
\end{abstract}

A diffusion process is one of the most important and common physical phenomena of nature. Coherent structures, like crystals and structural elements of biomolecules may shrink or grow gradually and randomly in a way that resembles a diffusive motion of their boundaries. On the one hand, inhomogeneities in a particular structure may disappear or be resolved into neighboring structures due to diffusion. On the other hand, diffusion typically tends to make the largest of the coherent structures larger. In contrast to this gradual modification of individual fragments, one might in addition encounter a completely different and abrupt physical phenomena, namely that of fragmentation. Fragmentation occurs in for example growing ice crystals, when the diffusively growing crystal is subjected to stresses that can cause a piece to break off, thus leading to two individual crystals, each of them continuing the competitive process between diffusion and fragmentation.

We believe that such interplay between gradual diffusion and rare but drastic fragmentation is a very common phenomenon in nature. In this letter we derive a general rate equation for the dynamical evolution of the density of fragments of a given size. The rate equation consists of a local diffusion term and non-local fragmentation terms. Differentiating once we obtain a third order differential equation in the size distribution of fragments. We solve the equation exactly by means of an eigenvalue expansion in terms of Bessel functions. The different eigenvalues correspond to different decay times such that in the infinite time limit we obtain the stationary distribution, corresponding to zero eigenvalue. This distribution is characterized by one parameter (the ratio between the diffusion and the fragmentation constants) and constitutes a universal stationary distribution for these competing processes. We apply experimental data from two very different physical processes, the size distribution of ice crystals in the Greenland ice sheet [1] and the length distribution of $\alpha$-helices in proteins of low homology. In both cases we find that the universal distribution fits the experimental data perfectly. In addition, for the ice crystals, we are

\footnotetext{
*email: borg,mhjensen,mathies,polesen,sneppen@nbi.dk
}

able to map out the entire dynamics of ice crystals 2000 years back in time [1].

In order to clarify the description, we consider a onedimensional simplification of the process in which the sizes of for instance ice-crystal grains are projected onto the coordinate $\tilde{x}$. The mechanism we propose then is described in terms of the density $N(\tilde{x}, \tilde{t})$ of "objects" (e.g. ice crystals or $\alpha$-helices in proteins) of a given length $\tilde{x}$ at a given time $\tilde{t}$. At a given time it is possible to increase or decrease $N(\tilde{x}, \tilde{t})$ by diffusion characterized by the diffusion constant $D$. Also, $N(\tilde{x}, \tilde{t})$ can be altered by fragmentation of the "objects" characterized by a fragmentation constant $f$, defined as the average number of break-ups in a time interval $d \tilde{t}$ over a length $L$. Thus, the number of fragments is given by $f L d \tilde{t}$. The constants $D$ and $f$ can depend on temperature and on quantities which are characteristics of the "objects". The resulting equation is

$$
\frac{\partial N(\tilde{x}, \tilde{t})}{\partial \tilde{t}}=D \frac{\partial^{2} N(\tilde{x}, \tilde{t})}{\partial \tilde{x}^{2}}-f \tilde{x} N(\tilde{x}, \tilde{t})+2 f \int_{\tilde{x}}^{\infty} d \tilde{x}^{\prime} N\left(\tilde{x}^{\prime}, \tilde{t}\right)
$$

Note that by this equation, the total "mass" of the fragments $\int_{0}^{\infty} \tilde{x} N(\tilde{x}, \tilde{t}) d \tilde{x}$ is conserved at any time in the evolution. The first term on the right hand side represents diffusion, the second term gives the fragmentation of objects with length $\tilde{x}$, and the last term is the contribution from fragmentation of objects larger than $\tilde{x}$. The factor of two in the last term follows formally from the condition of mass conservation, and results from the fact that there are two distinct ways to cut an object of length $\tilde{x}+\tilde{x}^{\prime}$ into segments of length $\tilde{x}$ and $\tilde{x}^{\prime}$. Similar approach has been applied in [2] for the stochastic dynamics of microtubules [3].

We introduce the dimensionless variables $x=\tilde{x} / \tilde{x}_{0}$ and $t=\tilde{t} / \tilde{t}_{0}$, where

$$
\tilde{t}_{0}=\left(D f^{2}\right)^{-1 / 3} \text { and } \tilde{x}_{0}=(D / f)^{1 / 3} .
$$

This amounts to considering eq. (II) with $D=f=1$, which we shall do in the following. We now differentiate eq. (11) with respect to $x$ and obtain

$$
\partial_{t} \partial_{x} N(x, t)=\partial_{x}^{3} N(x, t)-3 N(x, t)-x \partial_{x} N(x, t) .
$$

This equation can be solved by separation, using the boundary condition that $N(x, t) \rightarrow 0$ for $x \rightarrow \infty$. The 
result can be expressed in terms of the Airy function

$$
A(x)=\int_{0}^{\infty} d k \cos \left(k x+\frac{k^{3}}{3}\right),
$$

which in turn can be expressed in terms of Bessel functions 4],

$$
\begin{aligned}
& A(x)=\sqrt{\frac{x}{3}} K_{\frac{1}{3}}\left(\frac{2 x^{3 / 2}}{3}\right) \text { for } x>0 \\
& =\frac{\pi}{3} \sqrt{|x|}\left[J_{\frac{1}{3}}\left(\frac{2|x|^{3 / 2}}{3}\right)+J_{-\frac{1}{3}}\left(\frac{2|x|^{3 / 2}}{3}\right)\right] \\
& \text { for } x<0 .
\end{aligned}
$$

The solution of (3) is then

$$
N(x, t)=\sum_{n} C_{n} e^{\lambda_{n} t} B\left(x+\lambda_{n}\right), B(x)=-\partial_{x}^{2} A(x),
$$

where the sum may be an integral in case the eigenvalues $\lambda_{n}$ are in a continuous range. The function $B(x)$ is thus related to the Airy function by the second eq. (6). One further has

$$
B(x)=-x A(x) .
$$

This follows from the second eq. (6) by performing two differentiations of the expressions (5) and by use of standard Bessel function recursion relations. For a discussion of the Airy function we refer to [4].

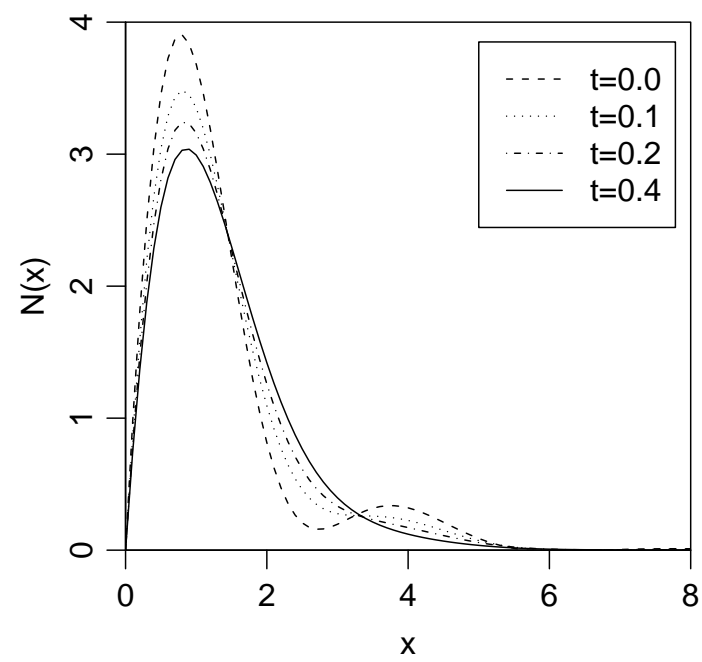

FIG. 1: An example showing the time development of $N(x, t)$ with a secondary peak, which ultimately disappears. The specific values of the expansion parameters $C_{n}$ are $\left(C_{0}, C_{1}, C_{2} \ldots\right)=(-7,-.2,-.2, .05,-.035)$.

We now impose the boundary condition that there should be no objects of size $x=0$,

$$
N(0, t)=0 \text {. }
$$

To implement the requirement (8) on the solution (6) we thus need that the eigenvalues $\lambda_{n}$ are the zeros of $B(x)$. From eq. (7) we see that $x=0$ is a zero. For $x>0$ there are no zeros, whereas for $x<0$ there exist an infinite number of zeros given as the solution of the equation

$$
J_{\frac{1}{3}}\left(\frac{2\left|\lambda_{n}\right|^{3 / 2}}{3}\right)+J_{-\frac{1}{3}}\left(\frac{2\left|\lambda_{n}\right|^{3 / 2}}{3}\right)=0 .
$$

These zeros can easily be found numerically. The first few are given approximately by $\lambda_{0}=0, \lambda_{1}=-2.338, \lambda_{2}=$ $-4.088, \lambda_{3}=-5.521, \lambda_{4}=-6.787, \lambda_{5}=-7.945, \lambda_{6}=$ -9.023 .

In order to find the the constants $C_{n}$ in the solution (6) we notice that the basic equation (3) is of third order, and hence does not lead to orthogonal functions. Instead we notice that from (7) the Airy function satisfies the second order differential equation,

$$
\partial_{x}^{2} A(x)=x A(x)
$$

for which standard methods are applicable: We define the auxiliary function

$$
M(x, t)=\sum_{n=0}^{\infty} C_{n} e^{\lambda_{n} t} A\left(x+\lambda_{n}\right),
$$

and we can then easily show from the second order differential equation (10) that the functions $A\left(x+\lambda_{n}\right)$ are orthogonal, and hence $C_{n}$ can be obtained in terms of an integral over the initial function $M(x, 0)$ times $A\left(x+\lambda_{n}\right)$. Since, however, we also have

$$
\partial_{x}^{2} M(x, t)=-N(x, t)
$$

from (6), the initial function $M(x, 0)$ can be expressed in terms of the corresponding initial function $N(x, 0)$, giving the result

$$
\begin{aligned}
C_{n} & =\frac{1}{I_{n}} \int_{0}^{\infty} d x A\left(x+\lambda_{n}\right) \\
& \times\left[\int_{x}^{\infty} d x^{\prime}\left(x-x^{\prime}\right) N\left(x^{\prime}, 0\right)-C_{0} A(x)\right],
\end{aligned}
$$

where

$$
\begin{aligned}
& I_{n}=\int_{0}^{\infty} d x A\left(x+\lambda_{n}\right)^{2}=A^{\prime}\left(\lambda_{n}\right)^{2} \\
& \text { and } C_{0}=\frac{-1}{A(0)} \int_{0}^{\infty} d x x N(x, 0) .
\end{aligned}
$$

This then allows us to write down the solution $N(x, t)$ for any given initial function $N(x, 0)$. A characteristic feature of this solution is that the mean value $\langle x\rangle$ saturates for large times. Using the solution one finds

$$
\langle x\rangle=\frac{\int_{0}^{\infty} x N(x, t)}{\int_{0}^{\infty} N(x, t)} \underset{t \rightarrow \infty}{\longrightarrow} \frac{3^{2 / 3} \Gamma(4 / 3)}{\Gamma(2 / 3)},
$$

which means that $\langle\tilde{x}\rangle \rightarrow 2.096(D / f)^{1 / 3}$. We also find that the dispersion saturates, $\left\langle x^{2}-\langle x\rangle^{2}\right\rangle \rightarrow 0.6074\langle x\rangle^{2}$, in 
marked contrast to pure diffusion, where the dispersion increases without bound. The time development of $N(x, t)$ is governed by the Boltzmann-like factors $\exp \left(\lambda_{n} t\right)$. In Fig. 1 we have shown by an example that these factors can give rise to additional secondary bumps, which ultimately disappear for larger times. It would be interesting to find actual examples where these structures are observed experimentally.

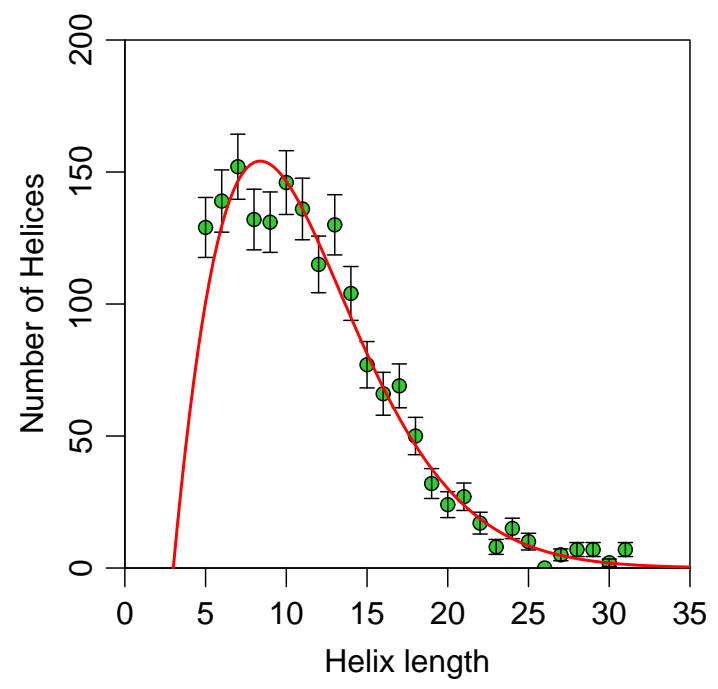

FIG. 2: The black dots represent the length distribution of $\alpha$-helices from 299 high resolution protein structures with low homology [5]. The error bars are estimated as the square root of the numbers. The full curve is the stationary distribution (i.e. infinite time limit) obtained from eq. (1) plotted versus the helix length measured in terms the number of amino acids. The ratio between the diffusion constant $D$ and the fragmentation rate $f$ resulting in the best fit to the data is $\left(\frac{D}{f}\right)^{1 / 3} \approx 6.1$ number of amino acids. Note that $N(x)$ vanishes for a helix length equal to 3 , as a $\alpha$-helix needs one turn to be identified, see $[7]$.

We now describe two widely different application of the suggested interplay between gradual diffusion and sudden fragmentation. The examples are selected from the constraint imposed not so much by the generic nature of the two processes, but also by the requirement that random merging of structures should be insignificant. Further, the two examples are taken from cases where it is reasonable to assume that fragmentation occurs uniformly on the size axis $x$, as implicitly assumed in the basic eq. (11) of the process. The two experimental data sets behind our studies are respectively the length distribution of $\alpha$-helices in proteins, and the size distribution of ice crystals in ice sheets. In the former case we will be able to extract the ratio $\frac{D}{f}$ by performing a least squares fit of the stationary state solution and in the latter case we do an exponential fit of $\langle x\rangle(t)$ using the two leading terms in the solution, $\frac{\langle x\rangle_{\infty}}{1+\left(\frac{\langle x\rangle \infty}{\langle x\rangle_{0}}-1\right) e^{-\left(t-t_{0}\right) / \tau}}$. We then extract both
$D$ and $f$ from (2) and (15) and by noting that the characteristic time $\tau$ is related to the fragmentation by the second largest eigenvalue $\lambda_{1}$, we obtain $\tau=-1 /\left(\lambda_{1} f\right)[1]$.

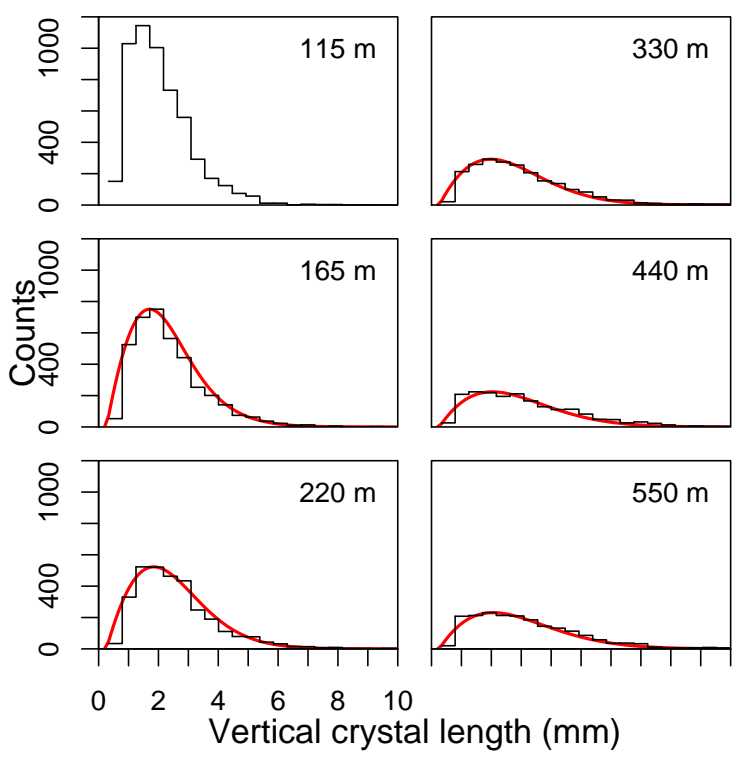

FIG. 3: Distributions of ice crystal sizes at depths $115 \mathrm{~m}, 165 \mathrm{~m}$, $220 \mathrm{~m}, 330 \mathrm{~m}, 440 \mathrm{~m}$ and $550 \mathrm{~m}$. The crystal size is defined as the vertical extension of the individual crystals. The ragged curves are the measured histograms and the smooth lines are the temporal evolution predicted by eq. (1) starting from the initial distribution at $115 \mathrm{~m}$. The total counts of ice crystals decreases with depth (due to the overall increase of sizes) until the steady state is reached. The values of the diffusion constant and the fragmentation rate used in these plots are $D=2.8 \cdot 10^{-3} \mathrm{~mm}^{2} /$ year and $f=3.6 \cdot 10^{-4} / \mathrm{cm} \cdot$ year. Note that the distributions vanish at a small, finite value to account for the experimental bias; see [1].

The length distribution of $\alpha$-helices 5 is taken from a database of 299 high resolution structures with low homology extracted from the PDB (Protein Data Bank) 5]. Secondary structure have been calculated on the basis of backbone hydrogen bonding [6] and in total 2101 helices are used [5]. Helices of lengths between 5 and 31 amino acids have been counted resulting in the distribution shown as circles in Fig. 22 Clearly, the distribution exhibits a maximum around a length of 7 amino acids followed by a long tail. The data do not fit well any simple statistical distribution and a polynomial fit requires at least a fourth order expression to become acceptable 5. We consider this distribution as the result of an 'infinitely' long evolutionary process where diffusive growth competes with intermittent fragmentation. The stationary solution to eq. (11) is defined by one free parameter (the ratio $D / f$ ) and it nicely reproduces the overall rise and decline of the observed helix frequency with length 7]. There are several points to make in this connection: 1) Considering ensembles of $\alpha$-helices, one effectively ran- 
domizes the particular evolutionary advantages of growing or shrinking any particular $\alpha$-helix in any particular protein. Thus whereas a particular change may have a purpose, then the overall process is probably well represented by random domain adjustments and occasional fragmentation. 2) Though $D$ and $f$ have essentially the same molecular origin, simple energy arguments suggest that a point mutation in the middle of the helix to a residue not forming hydrogen bonds has a statistical weight of $\approx 1 / 400$ relative to the weight of growing/shrinking the helix [8]. This corresponds to the ratio $(D / f)^{1 / 3} \approx 7.4$ in reasonable agreement with our result. 3) That in principle other processes also acts to limit the maximum length of $\alpha$-helices, as f.ex. the total size of proteins limits the maximal length of their sub elements. This maximum length will only influence the distribution of the very long $\alpha$-helices. Overall we see the resulting distribution of $\alpha$ helices as an ensemble average, revealing a process that leaves a statistical stationary distribution of structural elements in much the same way as binding energies between amino acids tend to influence the frequencies of neighboring relations between them in an ensemble of proteins 9].

For the ice crystal distribution, we apply data from the North Greenland Ice Core Project (NorthGRIP) which provides paleoclimatic information back to at least 115 kyr before present (B.P.) [10]. Each year, precipitation on the ice sheet covers it with a new layer of snow, which gradually transforms into ice crystals as the layer sinks into the ice sheet. The size distribution of ice crystals has been measured at selected depths in the upper 880 $m$ of the NorthGRIP ice core [1], which cover a time span of 5300 years [12]. Fig. 3] shows size distributions of ice crystals at selected depths down to $550 \mathrm{~m}$. The crystal size $x$ is defined as the vertical extent of a crystal [13]. Applying instead the horizontal extent of the crystal yields the same distribution curve thus supporting our assumption of using the linear size in our formalism.

Each distribution exhibits a pronounced peak, indicating a typical crystal size at each depth, followed by a decaying tail of relatively large crystals. The mean size becomes larger with depth and thus time until it saturates [14, 15]. The distributions gradually change with time toward a universal curve, indicating a common underlying physical process in the formation of crystals. We have identified this process as an interplay between the fragmentation of the crystals and the diffusion of their grain boundaries and is thus described by our general framework.

In this Letter we have presented a general evolutionary scenario for the dynamics of objects subjected to a competition between diffusion and fragmentation. The process results in an evolution equation of the density $N(x, t)$ of objects of linear size $x$. In the stationary limit, this equation predicts a "universal" distribution curve $N(x)$ determined only by the ratio $\frac{D}{f}$. This curve can be described exactly in terms of Bessel function leading to a power law uprise for small $x$ followed by a tail of a stretched exponential form for large $x\left(\exp \left(-x^{\frac{3}{2}}\right)\right)$. We speculate that this general competing principle might be the relevant mechanism in many other systems of nature and may result in distribution functions of a similar type.

We are grateful to Mogens Levinsen and Anders Svensson for discussions.
[1] J. Mathiesen, J. Ferkinghoff-Borg, M.H. Jensen, M. Levinsen, P. Olesen, D. Dahl-Jensen and A. Svensson, arXiv:physics/0310087

[2] H. Flyvbjerg, T. E Holy and S. Leibler, Phys. Rev. Lett. 73, 2372 (1994).

[3] The model proposed in 2] for the stochastic dynamics of microtubules focuses on transitions from the growing to the shrinking state of microtubules and contains a fragmentation term similar to the one in eq. (1). However, their work considers an open system and the model is therefore not mass conserving.

[4] G. N. Watson, A treatise on the Theory of Bessel Functions, Cambridge University Press (1944).

[5] Penel, S., Morisson, R. G., Mortishire-Smith, R. J. and Doig, A. J. J. Mol. Biol., 293, 1211-1219 (1999).

[6] Kabsch, W. and Sander, C., Biopolymers 22, 2577-2637 (1983).

[7] The boundary condition (8) has been replaced by $N\left(x_{0}, t\right)=0$, where $x_{0}=3$ in Fig. 2. In eq. (1) $x$ is replaced by $x-x_{0}$, since at least four amino acids are required to form an $\alpha$-helix, i.e. $x_{0}+1=4$.

[8] A. J. Doig, A. Chakrabartty, T. M. Klingler and R. Baldwin, Biochemistry 33, 3369 (1994).

[9] S. Miyazawa and R.L. Jernigen, Macromolecules 18, 534
(1985); S. Miyazawa and R.L. Jernigen, J. Mol. Biol. 256, $623(1996)$

[10] D. Dahl-Jensen, N. Gundestrup, H. Miller, O. Watanabe, S.J. Johnsen, J.P Steffensen, H.B. Clausen, A. Svensson, and L.B. Larsen, Annals of Glaciology 35, 1-5, 2002.

[11] A. Svensson, K.G. Schmidt, D. Dahl-Jensen, S.J. Johnsen, Y. Wang, J. Kipfstuhl, and T. Thorsteinsson, Annals of Glaciology 37 (2003).

[12] S.J. Johnsen, D. Dahl-Jensen, N. Gundestrup, J.P. Steffensen, H.B. Clausen, H. Miller, V. Masson-Delmotte, A.E. Sveinbjörndottir, and J. White, Journal of Quaternary Science 16 (4), 299-307, 2001.

[13] The model, eq (1), can be generalized by changing the fragmentation rate to be proportional to $x^{\beta}$ to account for energy/surface arguments. The stationary distribution can also be solved in these cases. For $\beta=2$ the solution will be $A(x) \sim \sqrt{x} K_{1 / 4}\left(x^{2} / 2\right)$ instead of eq. (5). However, this expression provides a poor fit to the experimental data for the ice crystals.

[14] T. Thorsteinsson, Berichte zur Polarforschung 205, 1-146 (1996).

[15] L. Jun, T.H. Jacka, and V. Morgan, Ann. Glaciology 27, 343-348 (1998). 